\title{
Hemosiderin in Decidua Parietalis
}

National Cancer Institute

\section{Source}

National Cancer Institute. Hemosiderin in Decidua Parietalis. NCI Thesaurus. Code

C118149.

The deposition of hemosiderin along the gravid uterine wall. 